\title{
Toll-like receptor 4 inhibition in lung ischemia-reperfusion injury: Time for a clinical trial?
}

\author{
Chadrick E. Denlinger, MD
}

See related article on pages 1653-61.

The toll-like receptors (TLRs) are a family of conserved cell-surface receptors, including at least 13 members present in all species ranging from Drosophila to mammals, that play a central role in the activation of the innate immune system. TLRs are primarily known to respond bacterial or viral epitopes. TLR4 has been recognized as a receptor for lipopolysaccharide from the outer membrane of gram-negative bacteria. In the setting of tissue injury such as ischemia-reperfusion injury (IRI), however, endogenous ligands activate an innate response mediated through TLR4. These ligands include heat shock protein, nonhistone chromatin-binding protein high-mobility group box 1 , and extracellular matrix proteins such as fibronectin, hyaluronan, heparin sulfate, and biglycan. ${ }^{1}$ For this reason, selective targeting of TLR4 is an attractive approach to mitigating IRI after solid organ transplantation.

Activation of TLR4 leads to dimerization of these transmembrane ligand receptors and the downstream activation of numerous inflammatory signaling pathways that include nuclear factor $\kappa \mathrm{B}$, Jun $\mathrm{N}$-terminal kinase, p38, and extracellular signal-regulated kinase 1/2. The Mulligan laboratory has previously published extensively regarding the roles of alveolar macrophages, lipopolysaccharide, and TLR4 in lung IRI., ${ }^{2,3}$ In their most recent publication, Phelan and colleagues ${ }^{4}$ have clarified the importance of TLR4 but not TLR2 in alveolar macrophage-mediated IRI. Conversely, TLR2 appeared more related to pulmonary artery endothelial cells and type 2 pneumocytes, and its inhibition had minimal impact on lung injury. Recognizing the specificity of TLRs in IRI may facilitate precise targeting of the signaling pathway most responsible for this process while preserving a greater part of the immune system. Importantly, in vivo experiments used systemic administration of TLR4 small

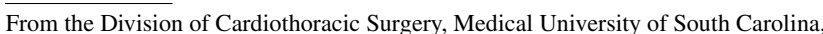
Charleston, SC.

Disclosures: Author has nothing to disclose with regard to commercial support.

Received for publication Feb 26, 2015; accepted for publication Feb 26, 2015; available ahead of print March 29, 2015.

Address for reprints: Chadrick E. Denlinger, MD, 25 Courtenay Dr, ART Suite 7018, Charleston, SC 29425 (E-mail: denlinge@musc.edu).

J Thorac Cardiovasc Surg 2015;149:1662-3

$0022-5223 / \$ 36.00$

Copyright (c) 2015 by The American Association for Thoracic Surgery

http://dx.doi.org/10.1016/j.jtcvs.2015.02.057 interfering RNA (siRNA), which targeted alveolar macrophages and limited lung permeability, a surrogate marker for IRI. This is not a significant leap from a clinically translatable strategy.

Previously, a major limitation for the broad application of siRNA therapy for numerous disease conditions has been a relative lack of effective delivery vehicles. The efficacy of direct intravenous injection of naked siRNA is limited by the fact that its small size contributes to rapid renal clearance, giving these compounds a half-life of 30 minutes. ${ }^{5}$ On the other hand, the molecules are too large and negatively charged to be readily absorbed directly by cells. Additional barriers to directed delivery of siRNA are opsonization of the compound and phagocytosis by the reticuloendothelial system, binding to the extracellular matrix, and off-target cellular uptake. Nevertheless, significant progress has been made in these respects. Modifications to the RNA itself, as well as encapsulation within liposomes or "stealth particles," have dramatically improved siRNA delivery, and this has paved the way for at least 17 different clinical trials with siRNA to treat conditions including macular edema, hypercholesterolemia, and acute kidney injury. ${ }^{5}$

Small-molecule inhibitors of TLR signaling cascades are also available. Matsunaga and colleagues ${ }^{6}$ recently described the use of TAK-242 as a small molecule that specifically binds the intracellular domain of TLR4 and blocks its interactions with downstream adaptor molecules. TAK-242 has been investigated clinically in a prospective, randomized study to determine the safety and efficacy of its use in patients with sepsis. ${ }^{7}$ Two hundred seventy-four patients with severe sepsis were randomly allocated to receive a placebo or 1 of 2 different doses of TAK-242, with the primary end point of all-cause mortality at 28 days. Modest trends that did not achieve clinical significance were found among patients treated with the higher dose of TAK-242, and the differences in survival appeared to be greatest among patients with both shock and respiratory failure. This study indicates that smallmolecule inhibition of TLRs may be clinically available in the near future.

As with most intracellular signaling pathways, there are numerous redundancies that may mitigate the effect of selective inhibition of a single molecule (Figure 1). Extracellular coupling molecules are required to activate TLR signaling driven by exposure to lipopolysaccharide and endogenous ligands released after tissue injury. An even greater number of second messengers are necessary to 


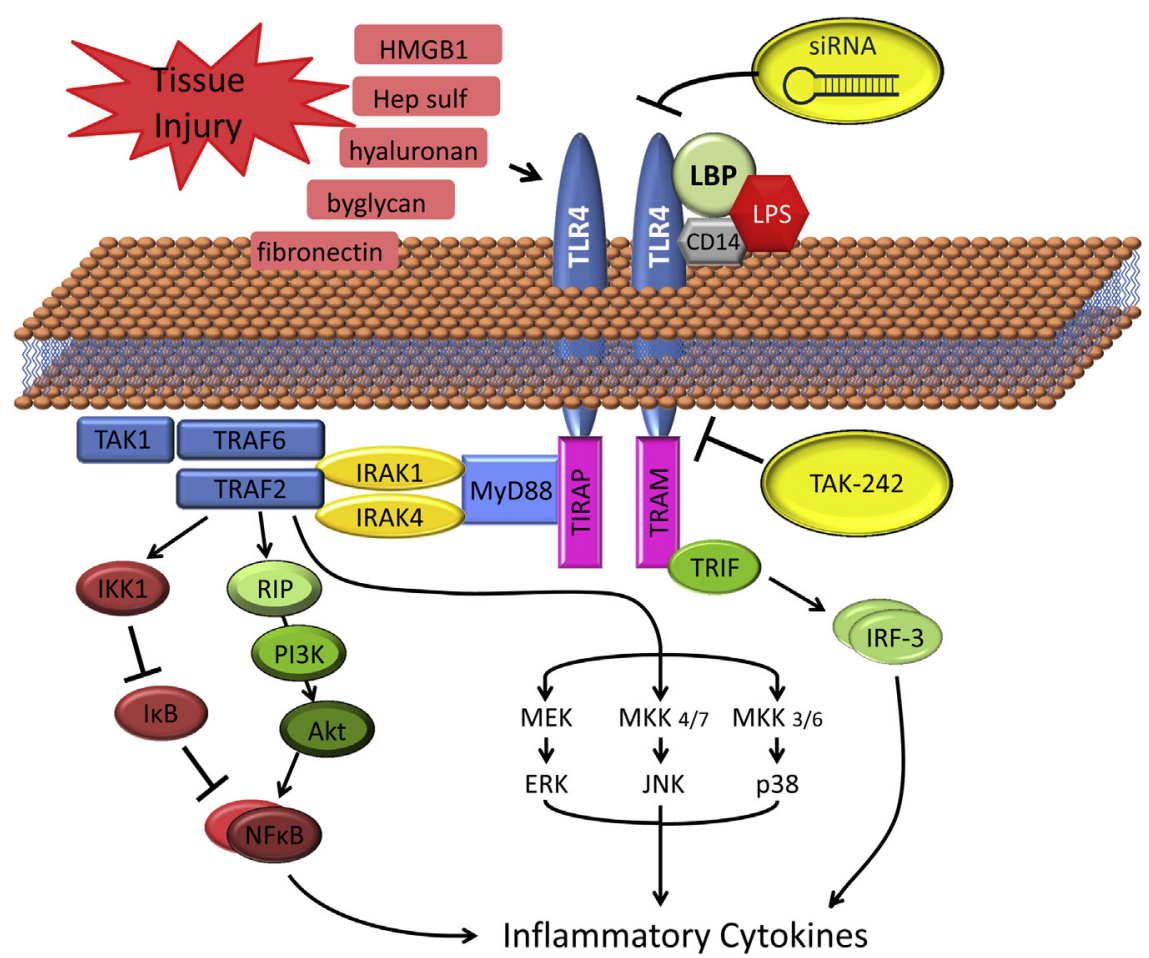

FIGURE 1. The signaling pathway in lung ischemia-reperfusion injury includes numerous redundancies that may mitigate the effect of selective inhibition of a single molecule; however, toll-like receptor 4 (TLR4) may act as a sort of molecular bottleneck. HMGB1, High-mobility group box 1; siRNA, small interfering RNA; Hep sulf, heparin sulfate; $L B P$, lipopolysaccharide-binding protein; $L P S$, lipopolysaccharide; TAK1, transforming growth factor $\beta$-activated kinase 1;TRAF, tumor necrosis factor receptor-associated factor; IRAK, interleukin 1 receptor-associated kinase; MyD88, myeloid differentiation factor 88; TIRAP, toll-interleukin 1 receptor domain-containing adapter protein; TRAM, TRIF-related adaptor molecule; TAK-242, investigational drug name; $I K K, \mathrm{I} K \mathrm{~B}$ kinase; $R I P$, receptor-interacting protein; TRIF, toll-interleukin receptor domain-containing adapter inducing interferon $\beta ; P I 3 K$, Phosphoinositide 3 kinase; $I R F 3$, interferon regulatory factor $3 ; I K B$, inhibitor of $\kappa \mathrm{B} ; A k t$, protein kinase $\mathrm{B}$; $M E K$, mitogen-activated protein kinase/extracellular signal-related kinase kinase; $M K K$, mitogen-activated protein kinase kinase; $E R K$, extracellular signal-regulated kinase; $J N K$, Jun N-terminal kinase; $N F \kappa B$, nuclear factor $\kappa \mathrm{B} ; C D 14$, cluster differentiation 14 .

convey the intracellular signals. Recognition of TLR4 as a molecular bottleneck in the signaling cascade of IRI may indicate that it should be the most attractive target.

In their article in this issue of the Journal, Erridge and colleagues have demonstrated the critical role for TLR4 in IRI after lung transplantation and have shown that selective inhibition of TLR4 with siRNA in an in vivo model greatly attenuates this injury. These findings are very important because of the high incidence of IRI and the closeness to clinical availability of siRNA or small-molecule treatment strategies for selective inhibition of TLR4.

\section{References}

1. Erridge C. Endogenous ligands of TLR2 and TLR4: agonists or assistants? J Leukoc Biol. 2010;87:989-99.
2. Merry HE, Wolf PS, Fitzsullivan E, Keech JC, Mulligan MS. Lipopolysaccharide pre-conditioning is protective in lung ischemia-reperfusion injury. $J$ Heart Lung Transplant. 2010;29:471-8.

3. Naidu BV, Krishnadasan B, Farivar AS, Woolley SM, Thomas R, Van Rooijen N, et al. Early activation of the alveolar macrophage is critical to the development of lung ischemia-reperfusion injury. J Thorac Cardiovasc Surg. 2003;126:200-7.

4. Phelan P, Merry HE, Hwang B, Mulligan MS. Differential toll-like receptor activation in lung ischemia-reperfusion injury. J Thorac Cardiovasc Surg. 2015;149: 1653-61.

5. Zhou Y, Zhang C, Liang W. Development of RNAi technology for targeted therapy - a track of siRNA based agents to RNAi therapeutics. J Control Release. 2014:193:270-81

6. Matsunaga N, Tsuchimori N, Matsumoto T, Ii M. TAK-242 (resatorvid), a smallmolecule inhibitor of toll-like receptor (TLR) 4 signaling, binds selectively to TLR4 and interferes with interactions between TLR4 and its adaptor molecules. Mol Pharmacol. 2011;79:34-41.

7. Rice TW, Wheeler AP, Bernard GR, Vincent JL, Angus DC, Aikawa N, et al. A randomized, double-blind, placebo-controlled trial of TAK-242 for the treatment of severe sepsis. Crit Care Med. 2010;38:1685-94. 